\title{
Recognizing and Treating Valproic Acid Toxicity: A Case Report
}

\author{
John B. Crudup III ${ }^{\mathrm{a}, \mathrm{b}}$, Bryan I. Hartley ${ }^{\mathrm{a}}$, Bradley R. Keela, Mukta Panda ${ }^{\mathrm{a}}$
}

\begin{abstract}
Valproic Acid (VPA) toxicity has been steadily increasing in frequency since the FDA approved the drug for acute mania in 1995. According to the 2005 Annual Report of the American Association of Poison Control Center's Toxic Exposure Surveillance System (TESS), 8705 acute exposures to valproic acid occurred with major adverse outcomes reported in 404 patients of this cohort. Recognizing VPA toxicity is the first step in properly managing the patient. Key signs and symptoms of VPA toxicity include: CNS depression, lethargy and potential encephalopathy, respiratory depression, nausea/vomiting, and myoclonus. Lab abnormalities often include, in addition to elevated serum VPA levels, hypernatremia, elevated anion gap metabolic acidosis, hyperosmolality, hypocalcemia, and hyperammonemia with a transaminitis. A non-contrast head CT may demonstrate cerebral edema, the peak occurrence of which is between 12 hours and 4 days after ingestion. Activated charcoal can be given early if the timing of overdose is known. Serial head CT scans should be ordered to monitor the possible delayed side effect of cerebral edema. Other areas of treatment deal with correcting the ill effects of the metabolic abnormalities (e.g. replace calcium if hypocalcemia). Specifically, lactulose is used to decrease serum ammonia levels and reduce the risk of encephalopathy. Another mainstay of treatment, L-carnitine, increases levels of free carnitine to participate in mitochondrial fatty acid synthesis. In extreme cases, hemodialysis may be required to reduce the levels of VPA and the associated metabolites. Of note our patient presented with virtually all the lab abnormalities and symptoms typical of VPA toxicity. He improved with hemodialysis and was discharged to a mental health facility in stable condition. Though VPA toxicity occurs rarely, it has a consistent presentation, symptomotology and defined treatment regimen. Awareness of a typical patient's presentation would
\end{abstract}

Manuscript accepted for publication June 9, 2011

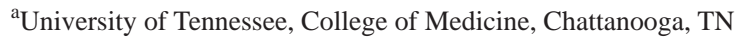
37403, USA

${ }^{\mathrm{b}}$ Corresponding author: John B. Crudup III, UT College of Medicine Chattanooga 975 East Third Street, Box 94 Chattanooga, USA.

E-mail: jbcrudup0308@gmail.com

doi:10.4021/jmc234w assist in early diagnosis and a successful treatment.

Keywords: Valproic Acid; Toxicity

\section{Introduction}

Valproic acid (VPA), derived from valerian acid, is a branched-chain fatty acid primarily used as an anti-epileptic and mood stabilizer. The mechanism of action of VPA revolves around its ability to potentiate the effects of the neurotransmitter Gamma Amino Butyrate (GABA). Specifically, VPA inhibits GABA degradation as well as GABA Transaminobutyrate (ABAT), increases GABA synthesis, and decreases turnover. This in turn allows increased availability of GABA at the synapse. Mild side effects of VPA include decreased sleep at the end of treatment, weight gain, drowsiness, nausea, and unsteadiness. However, if taken in supra-therapeutic doses, VPA can lead to toxicity and have a significant impact on patient's safety. VPA toxicity includes signs and symptoms of CNS depression, lethargy, encephalopathy, and respiratory depression [1].

Valproic acid toxicity has been steadily increasing in frequency since the FDA approved the drug for acute mania in 1995. According to the 2008 Annual Report of the American Association of Poison Control Center's National Poison Data System (NPDS), 8,705 acute exposures to valproic acid occurred. Of these cases, major adverse outcomes were reported in 404 patients of this cohort [2].

Valproic acid toxicity though rare is an easily identifiable clinical scenario. When encountered, it has a typical presentation that can be dealt with promptly if the care giver has a baseline level of knowledge about the presenting signs and symptoms. The goal of this case report is to help explain and educate clinicians on the presentation and the treatment of VPA toxicity.

\section{Case Report}

A 30-year-old white male with a history of hypertension and 
bipolar disorder presented to an outside hospital after his wife heard him fall from his bed. At this time, she found him unarousable. Prior to this event he was in his normal state health. At the outlying facility he was confused and combative with a Glasgow Coma Scale (GCS) of 3. He was therefore transferred to our institution for a higher level of care. In route, he was intubated due to apneic episodes and further airway protection. On arrival vitals were stable, he was obtunded, withdrew all extremities to pain, and had a gag and corneal reflex with bilateral lower extremity clonus noted. Initial information was that the patient had overdosed on some of his depression medications. It was then learned that the medication was valproic acid.

Multiple laboratory values were obtained, and significant derangements noted were: anion gap metabolic acidosis of 23, hypernatremia (153 mmol/L), hypocalcemia $(7.8 \mathrm{mg} /$ $\mathrm{dl})$, hyperosmolality (321 mmol/kg), and hyperammonemia (176 umol/L). Computerized tomography (CT) of the head showed no acute abnormalities. The patient's VPA level was found to be $990 \mathrm{mcg} / \mathrm{mL}$ (nml 50-100 mcg/mL).

Initially poison control was notified and supportive measures were begun. The patient was placed in the ICU for continuous monitoring and nephrology was consulted for evaluation for hemodialysis. Nephrology's opinion was that he could benefit from dialysis. The patient underwent one treatment of hemodialysis and had dramatic improvement of his VPA blood levels from $998 \mathrm{ug} / \mathrm{mL}$ to $207 \mathrm{ug} / \mathrm{mL}$. At this point, the patient was still unresponsive. Because of concern for cerebral edema, a repeat CT of the head was performed and again showed no acute abnormalities. The patient remained on a ventilator overnight.

The morning after admission his VPA level had been reduced to $90 \mathrm{ug} / \mathrm{mL}$ and his dialysis access was removed. A chest $\mathrm{x}$-ray was peformed and showed a right lower lobe infiltrate. Because of this, the patient required continued ventilator support and antibiotic coverage for a presumed aspiration pneumonia. By hospital day three, his mental status showed some improvement and he was extubated. He continued to become more alert and was transferred out of the ICU on day four of admission. On day five, the patient was doing well and back to his baseline mental status. He was discharged to a psychiatric facility for management of his psychiatric issues including suicidal ideation and bipolar disorder.

\section{Discussion}

Recognizing VPA toxicity is the first step in appropriate management. Although rare, literature reveals a consistent presentation involving specific metabolic and structural derangements. Key signs and symptoms include: CNS depression, lethargy and encephalopathy, respiratory depression, nausea/vomiting and myoclonus. Laboratory abnormalities are particularly helpful in this case. In addition to elevated VPA levels, blood chemistries often demonstrate hypernatremia, elevated anion gap metabolic acidosis, hyperosmolality, hypocalcemia, and hyperammonemia with a transaminitis. Helpful imaging studies include noncontrast head CT that may demonstrate cerebral edema, the peak of which occurs between 12 hours and 4 days after ingestion [3, 4].

Biochemical knowledge behind VPA toxicity allows an understanding of the associated metabolic abnormalities. The hypernatremia is due to VPA's composition: sodium valproate (approximately $13.8 \mathrm{mg}$ sodium/100 mg valproate) [5]. The hyperosmolality and anion gap acidosis result from elevated VPA metabolites which are organic anionic acids (propionic acid derivatives). These are osmotically active, leading to hyperosmolality and contribute to anion gap acidosis. Further, VPA metabolites are negatively charged, which facilitate binding to free ionized calcium causing hypocalcemia [3]. The propionic acid derivatives increase ammonia levels by two key mechanisms: first, they inhibit the hepatic mitochondrial enzyme carbamyl phosphate synthetase, the first step in eliminating ammonia during the urea cycle [6]; second, metabolites interfere with carnitine, another mitochondrial co-factor which also increases ammonia levels $[7,8]$.

The treatment of VPA toxicity is primarily supportive. Activated charcoal can be given early if the timing of overdose is known, especially if sustained release forms have been ingested sometimes several doses of activated charcoal can be helpful [9]. VPA is absorbed rapidly so if timing of ingestion is unknown this may not be helpful. In patients with CNS depression and unknown timing, the use of charcoal is limited since the risk of an aspiration event would be increased. Also, the treatment with charcoal will not change supportive measures or decrease plasma concentrations of VPA or its metabolites. Serial head CT scans may be needed to monitor the side effect of cerebral edema. Cerebral edema in VPA toxicity can have delayed onset. This edema is thought to be precipitated by the abnormal metabolism of VPA that allows accumulation of 2-EN-VPA in the brain and plasma. This metabolite has a long half life (around 43 hours), thus accounting for the potentially delayed onset of cerebral edema $[3,5]$.

Calcium should be replaced to avoid arrhythmias. Lactulose is used to trap ammonium in the gut, decreasing serum ammonia levels and reducing the risk of encephalopathy. Another mainstay of treatment is L-carnitine. L-carnititine increases levels of free carnitine to participate in mitochondrial fatty acid synthesis [10]. In extreme cases, hemodialysis may be required to reduce the levels of VPA and the associated metabolites. Hemodialysis can filter off free VPA. At blood levels above 90-100, the protein binding sites become saturated and there is a progressive increase in the free serum drug levels, which can be easily filtered by hemodialysis [11]. Though VPA toxicity occurs rarely, it has a consistent 
presentation and defined treatment regimen. Awareness of a typical patient's presentation would assist in early diagnosis and successful treatment.

\section{Grant Support}

None

\section{References}

1. Chateauvieux S, Morceau F, Dicato M, Diederich M. Molecular and therapeutic potential and toxicity of valproic acid. J Biomed Biotechnol. 2010;2010.

2. Bronstein AC, Spyker DA, Cantilena LR, Jr., Green JL, Rumack BH, Giffin SL. 2008 Annual Report of the American Association of Poison Control Centers' National Poison Data System (NPDS): 26th Annual Report. Clin Toxicol (Phila). 2009;47(10):911-1084.

3. Dupuis RE, Lichtman SN, Pollack GM. Acute valproic acid overdose. Clinical course and pharmacokinetic disposition of valproic acid and metabolites. Drug Saf. 1990;5(1):65-71.
4. Khoo SH, Leyland MJ. Cerebral edema following acute sodium valproate overdose. J Toxicol Clin Toxicol. 1992;30(2):209-214.

5. Gram L, Bentsen KD. Valproate: an updated review. Acta Neurol Scand. 1985;72(2):129-139.

6. Coulter DL, Allen RJ. Secondary hyperammonaemia: a possible mechanism for valproate encephalopathy. Lancet. 1980;1(8181):1310-1311.

7. Ishikura $\mathrm{H}$, Matsuo $\mathrm{N}$, Matsubara $\mathrm{M}$, Ishihara $\mathrm{T}$, Takeyama N, Tanaka T. Valproic acid overdose and L-carnitine therapy. J Anal Toxicol. 1996;20(1):55-58.

8. Gidal BE, Inglese CM, Meyer JF, Pitterle ME, Antonopolous J, Rust RS. Diet- and valproate-induced transient hyperammonemia: effect of L-carnitine. Pediatr Neurol. 1997;16(4):301-305.

9. Graudins A, Aaron CK. Delayed peak serum valproic acid in massive divalproex overdose--treatment with charcoal hemoperfusion. J Toxicol Clin Toxicol. 1996;34(3):335-341.

10. Ohtani Y, Endo F, Matsuda I. Carnitine deficiency and hyperammonemia associated with valproic acid therapy. J Pediatr. 1982;101(5):782-785.

11. Klotz U, Antonin KH. Pharmacokinetics and bioavailability of sodium valproate. Clin Pharmacol Ther. 1977;21(6):736-743. 